\title{
VALIDATION OF SHIP MANOEUVRING IN SHALLOW WATER THROUGH FREE- RUNNING TESTS
}

\author{
Katrien Eloot \\ Flanders Hydraulics Research, Antwerp, Belgium \\ Ghent University, Ghent, Belgium
}

\author{
Marc Vantorre \\ Ghent University \\ Ghent, Belgium
}

\author{
Guillaume Delefortrie \\ Flanders Hydraulics Research \\ Antwerp, Belgium \\ Frans Quadvlieg \\ MARIN \\ Wageningen, the Netherlands
}

\begin{abstract}
The shallow water effect on ship manoeuvring cannot be neglected. Most sea-going ships become more course stable when they sail from deep to (very) shallow water. International collaborations such as SIMMAN intend to grade up the knowledge on ship manoeuvring prediction through model tests and system based and numerical methods. Free-running model tests executed with the very large crude carrier KVLCC2 at two laboratories have been compared with the results of simulated turning circles and zigzag manoeuvres from two different mathematical models. It was concluded that the type of mathematical model has an important influence on the simulated behaviour. Moreover, further research is necessary as simulations result into a more course stable behaviour compared to free-running tests at model scale.
\end{abstract}

\section{INTRODUCTION}

The prediction of shallow water manoeuvring is still treated in a stepmotherly way in ship hydrodynamics as can be seen on pilot cards and wheelhouse posters where ship manoeuvring is illustrated based on estimated turning circles in shallow water. The background of these estimated manoeuvring results is not always clear and often based on empirical formulae to incorporate the shallow water effect in the simulation models.

To improve the knowledge on ship manoeuvring in shallow water, international workshops and projects as the SIMMAN workshop (http://www.simman2014.dk/) and the SimVal project (http://www.sintef.no/Projectweb/SimVal/) group research institutes worldwide to study the prediction of ship manoeuvring based on model tests, numerical methods and full scale trials.

The paper focuses on the validation process through model testing executed at Flanders Hydraulics Research (FHR), a Flemish governmental research institute that established the Knowledge Centre for Manoeuvring in Shallow and Confined Water together with Ghent University (www.shallowwater.be). In the framework of the SIMMAN workshop, captive and freerunning model tests have been executed with the benchmark very large crude carrier KVLCC2 in medium deep and (very) shallow water with under keel clearances (UKC) as low as $20 \%$. The influence of water depth as implemented in mathematical manoeuvring models has been validated based on free-running model tests executed in the Maritime Research Institute Netherlands (MARIN) with exactly the same ship model of the KVLCC2 used by FHR but with a slightly different propeller. Differences can be seen between the results from simulated turning circles and zigzag manoeuvres and the free-running characteristics of the same manoeuvring trials. Scale effects can be neglected as all test results are on model scale, but the testing technique (captive or free-running) and the mathematical model formulation will influence the predictive power of the simulation. Therefore a comparison is made between an empirical model of MARIN and a PMM test program based tabular model of FHR.

The validation process shows that hull, propeller or rudder dependent coefficients must be tuned to meet the free-running test results where the tuning additionally depends on the loading condition and the water depth to draft ratio or UKC.

\section{FREE-RUNNING AND CAPTIVE MODEL TESTS}


The execution of free-running and captive model tests for the prediction of the manoeuvring behaviour of different types of vessels is a common practice but often not fully considered due to the high cost of these tests. Nevertheless, the shallow water effect is not thoroughly understood so that these tests help in determining the hydrodynamic forces and moments on the ship model in captive mode and the overall behaviour due to propeller and rudder action in free-running mode.

At FHR a full manoeuvring data set consists of 300 captive model tests for one loading condition and one UKC. This number can be easily executed in ten calendar days with the fully automated Computerised Planar Motion Carriage (CPMC, [1]). The tests predict the manoeuvring behaviour in the four quadrants of operation of the ship (ship velocity and propeller rpm). The SIMMAN benchmark data set for the KVLCC2 was especially designed for the first quadrant of operation (forward speed and positive propeller rpm) and consisted of 97 captive model tests. The drift angle range was kept below 12 degrees so that the model is not valid for the prediction of forces and moments at large drift angles.

\subsection{MODEL CHARACTERISTICS}

The characteristics of the KVLCC2 are shown in Table 1. The KVLCC2 has been tested at an identical draft and up to three UKCs in captive and free-running mode both at MARIN (only free-running, $\mathrm{h} / \mathrm{T}=1.2$ and $1 / 5$ ) and FHR (captive: $\mathrm{h} / \mathrm{T}=$ 1.2, 1.5 and 1.8 and free-running: $\mathrm{h} / \mathrm{T}=1.2$ ). This one-to-one validation, with the same conditions in free-running and captive mode, helps in limiting the varying parameters during testing and modelling. Nevertheless based on the tank characteristics discussed in chapter 2.2 not all test parameters could be chosen identical in free-running mode at FHR and MARIN.

Table 1 -Full scale parameters of the KVLCC2

\begin{tabular}{|c|c|c|}
\hline $\mathrm{SC}$ & 75 & Scale \\
\hline $\mathrm{L}_{\mathrm{OA}}$ & 325.5 & $\mathrm{~m}$ \\
\hline $\mathrm{L}_{\mathrm{PP}}$ & 320 & $\mathrm{~m}$ \\
\hline $\mathrm{B}$ & 58 & $\mathrm{~m}$ \\
\hline $\mathrm{D}$ & 30 & $\mathrm{~m}$ \\
\hline $\mathrm{T}_{\mathrm{F}}$ & 20.80 & $\mathrm{~m}$ \\
\hline $\mathrm{T}_{\mathrm{A}}$ & 20.80 & $\mathrm{~m}$ \\
\hline $\mathrm{T}_{\mathrm{M}}$ & 20.80 & $\mathrm{~m}$ \\
\hline $\mathrm{VOL}$ & 311600 & $\mathrm{~m}^{3}$ \\
\hline $\mathrm{C}_{\mathrm{B}}$ & 0.81 & \\
\hline \multicolumn{3}{|c|}{ Propeller } \\
\hline $\mathrm{Max} \mathrm{rpm}$ & 100 & \\
\hline $\mathrm{Dp}$ & 9.825 & $\mathrm{~m}$ \\
\hline $\mathrm{P} / \mathrm{Dp}$ & 0.721 & - \\
\hline $\mathrm{AEP}$ & 0.431 & - \\
\hline \multicolumn{3}{|c|}{ Rudder } \\
\hline $\mathrm{A}_{\mathrm{R}}$ & 111.7 & $\mathrm{~m}^{2}$ \\
\hline
\end{tabular}

The tested UKC values are presented in Table 2 for the KVLCC2. These UKCs vary between medium deep water, (100\%), shallow (50\%) and very shallow water (20\%) according to the PIANC classification [2] of water depth to draft ranges according to their effect on ship manoeuvrability.

For the KVLCC2 the focus will be on the (very) shallow water results as most comparative data are available for the $20 \%$ UKC case.

Table 2 - Under keel clearances for KVLCC2 in percentages of the draft

\begin{tabular}{|c|c|}
\hline & KVLCC2 \\
\hline UKC 1 & 80 \\
\hline UKC 2 & 50 \\
\hline UKC 3 & 20 \\
\hline
\end{tabular}

\subsection{TANK CHARACTERISTICS}

The tank characteristics and the bottom accuracy of the (towing) tanks of MARIN and FHR are summarized in Table 3. The bottom accuracy is within the $10 \%$ limit of UKC according to the International Towing Tank Conference [3].

Table 3 - Tank characteristics and bottom accuracy

\begin{tabular}{|c|c|c|c|c|c|c|}
\hline Institute & $\mathrm{L}$ & $\mathrm{W}$ & $\mathrm{h}_{\max }$ & $\mathrm{h} / \mathrm{T}$ & $\mathrm{h}$ & Bottom accuracy \\
\hline & {$[\mathrm{m}]$} & {$[\mathrm{m}]$} & {$[\mathrm{m}]$} & {$[-]$} & {$[\mathrm{m}]$} & {$[\%$ of UKC] } \\
\hline MARIN & 220 & 15.8 & 1.15 & 1.2 & 0.333 & $5.4 \%$ \\
\cline { 5 - 7 } & & & & 1.5 & 0.416 & $2.2 \%$ \\
\hline FHR & 68 & 7 & 0.5 & 1.2 & 0.333 & $1.8 \%$ \\
\hline
\end{tabular}

Each tank uses different release procedures for setting the initial conditions of the ship models in free-running mode:

- At MARIN the ship model is accelerated on its own, so that the initial conditions cannot be controlled exactly and tests are repeated to choose the most appropriate test.

- At FHR the ship model is accelerated in captive mode by the carriage (Figure 1). Once the initial conditions are met, the ship model is released and runs in freerunning mode.

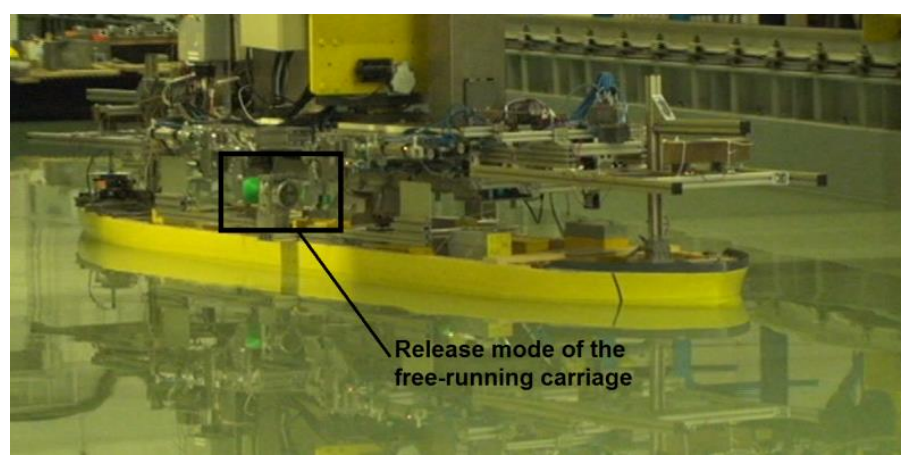


Figure 1 - Release mode of the carriage for free-running at FHR

All free-running manoeuvres have been executed with constant RPM. Due to the large difference in length and width of the tank at MARIN compared to the tank at FHR the blockage (ratio of ship model section to tank section) differs and influence of tank walls can be expected.

\subsection{TURNING CIRCLES}

Due to the restricted width of both tank facilities, only partial turning circles could be executed, limited to 90 degrees course change at MARIN and to about 40 degrees at FHR as shown in Figure 2.

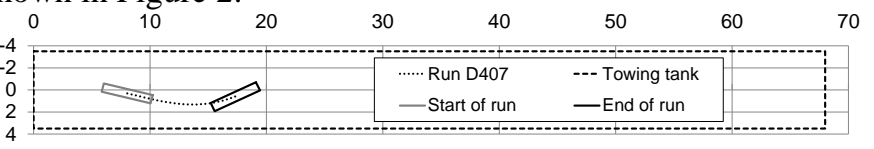

Figure 2 - Partial turning circle manoeuvre at FHR

For the execution of an uncertainty analysis the SIMMAN committee requested the execution of several (ten) runs. Some of these runs from FHR and MARIN are shown in Figure 3 (in prototype, i.e. full scale). Run 3 and 4 from FHR and 1 and 5 of MARIN come close to each other with comparable paths although other runs are more diverging for both institutes. The spread of all shown runs is indeed large and an uncertainty analysis should be executed. In chapter 4 the validation with the prediction results based on mathematical models will be executed for the runs 3 and 4 of FHR and run 1 for MARIN.

The time dependence of the rate of turn is summarized in Figure 4 for the selected runs of Figure 3. For all runs executed by MARIN an overshoot in the rate of turn compared to the steady state value is observed. The steady state is not necessarily obtained during the 90 degrees course change tests but are nevertheless considered and extrapolated as the steady value. The values measured in the tank of FHR during runs 1 and 2 differ considerably from these measured by MARIN and are therefore excluded from further analysis. The vicinity of the tank walls considerably influence the end of the test (no convergence to a steady state) and large diverging values are measured for the maximum rate of turn at FHR with values between -0.25 and $-0.3 \mathrm{deg} / \mathrm{s}$.

The initial conditions of the test runs of MARIN are not always characterized by a constant heading as the rate of turn can be up to $-0.05 \mathrm{deg} / \mathrm{s}$ at the start of the turn. Setting identical initial conditions was not possible with the release mode used by MARIN.

\subsection{ZIGZAG MANOEUVRES}

Zigzag manoeuvres were chosen as 10/2.5 and 20/5 due to the vicinity of the tank walls in the test facility of FHR. A clear difference is observed between the spread and phase shift for the 10/2.5 (Figure 5) compared to the 20/5 (Figure 6) zigzag tests at FHR. Thanks to the release mode at FHR the initial conditions can be exactly repeated which is not observed for all zigzag manoeuvres at MARIN (a non-zero rate of turn for run 3 and thus excluded from the analysis).

The larger reliability of the 20/5 tests is confirmed by the statistics summarized in

Table 4. The spread on the ten test runs of FHR is larger than what is observed in Figure 6 as only the four tests close to the average values were selected for presentation in Figure 5 and Figure 6. Among the four repeated test runs at MARIN especially run 3 is giving biased results for the first and second execute with a phase shift due to a non-zero rate of turn at the time of the start of the manoeuvre.

For the validation in chapter 4 , run 2 of FHR has been chosen for the $+10 /-2.5$ zigzag and run 7 of FHR for the $-20 /+5$ zigzag. For the latter run 1 of MARIN has been selected.

Table 4 - Statistics for the 10/-2.5 and -20/5 zigzags (20\% UKC) with KVLCC2 through free-running tests at FHR

\begin{tabular}{|c|c|c|c|c|c|c|c|}
\hline & & \multicolumn{3}{|c|}{ 10/-2.5 zigzag } & \multicolumn{3}{|c|}{$-20 / 5$ zigzag } \\
\hline SUMMARY & & $\begin{array}{l}\frac{1}{0} \\
\frac{1}{1} \\
\frac{\alpha}{2} \\
\frac{1}{2}\end{array}$ & 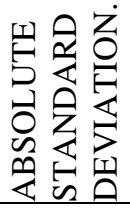 & 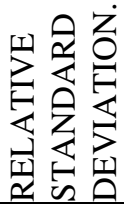 & $\begin{array}{l}\frac{1}{0} \\
\frac{\alpha}{4} \\
\frac{\alpha}{2} \\
\frac{1}{4}\end{array}$ & 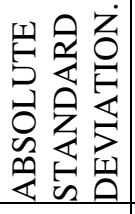 & 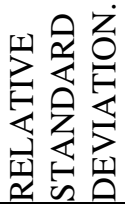 \\
\hline Period & $(\mathrm{s})$ & 58.554 & 2.550 & $4.35 \%$ & 55.807 & 0.897 & $1.61 \%$ \\
\hline Time till 1st heading deviation (HD) & $(\mathrm{s})$ & 11.286 & 1.071 & $9.49 \%$ & 8.924 & 0.242 & $2.71 \%$ \\
\hline Travelled distance till 1st HD & $(\mathrm{m})$ & 4.675 & 0.437 & $9.35 \%$ & 3.696 & 0.099 & $2.68 \%$ \\
\hline Max. heading 1 & $\left({ }^{\circ}\right)$ & -3.803 & 0.582 & $15.31 \%$ & 9.729 & 0.338 & $3.47 \%$ \\
\hline Max. heading 2 & $\left({ }^{\circ}\right)$ & 8.780 & 0.953 & $10.85 \%$ & -13.192 & 0.414 & $3.14 \%$ \\
\hline
\end{tabular}




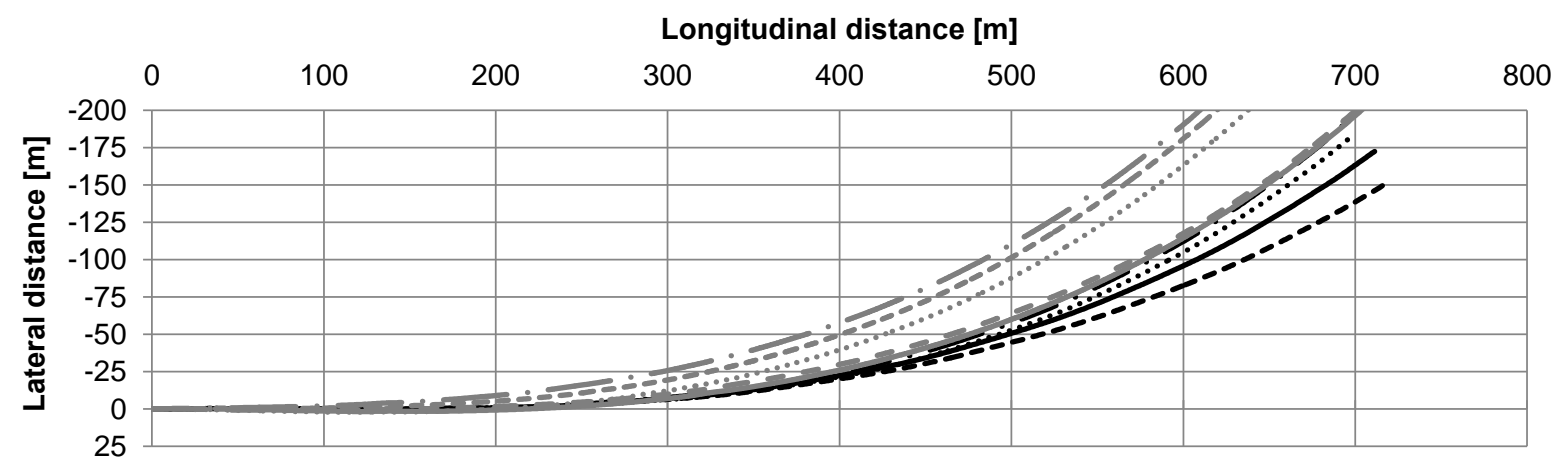

-FHR, Run 1
$\ldots$ - FHR, Run 2
$\ldots . .$.
FHR, Run 3
- FHR, Run 4
MARIN, Run 1
$\ldots$ - MARIN, Run 2
$\ldots . .$. MARIN, Run 3
- MARIN, Run 4
- MARIN, Run 5

Figure 3 - Paths for partial turning circles to port (20\% UKC) with KVLCC2 through free-running tests at FHR and MARIN, prototype values

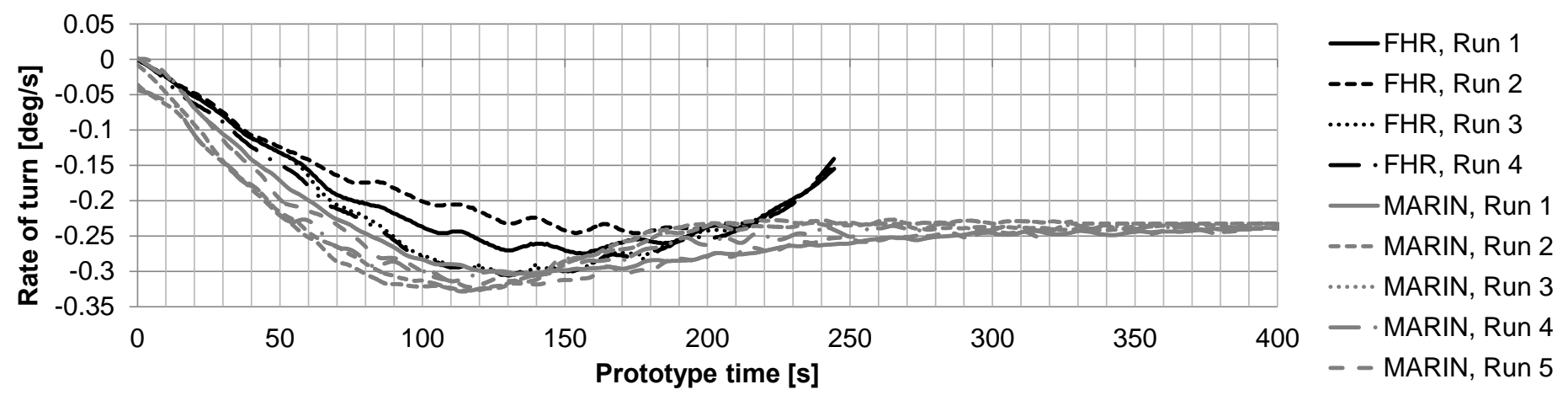

Figure 4 - Time dependence of the rate of turn for the partial turning circles to port (20\% UKC) with KVLCC2 through free-running tests at FHR and MARIN

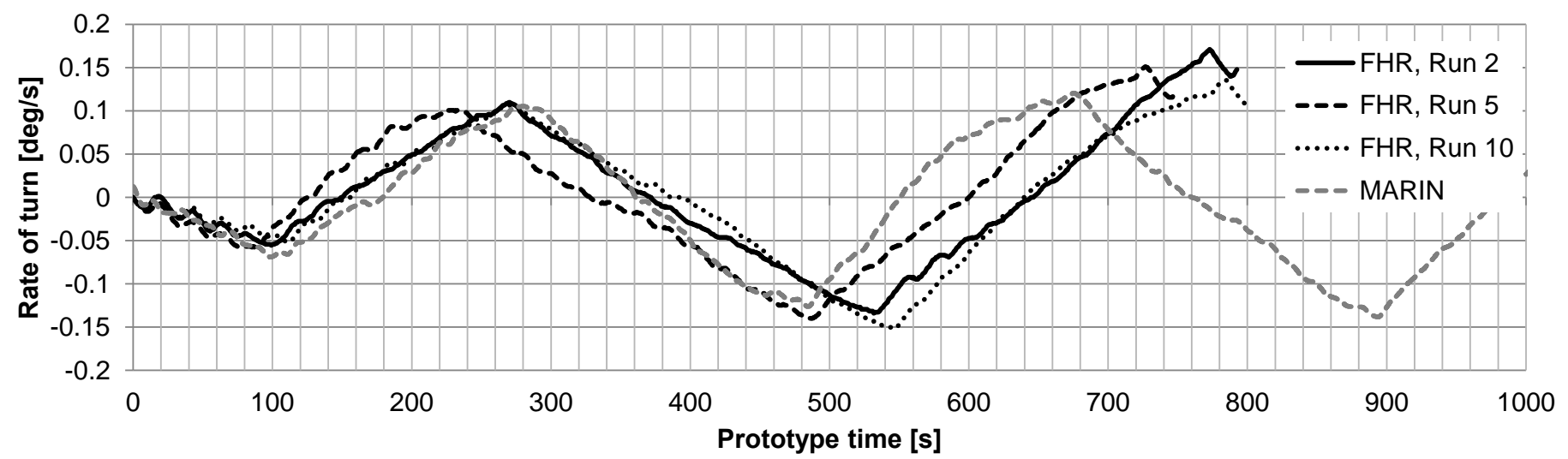

Figure 5 - Time dependence of the rate of turn for the +10/-2.5 zigzag manoeuvres (20\% UKC) with KVLCC2 through free-running tests at FHR and MARIN 


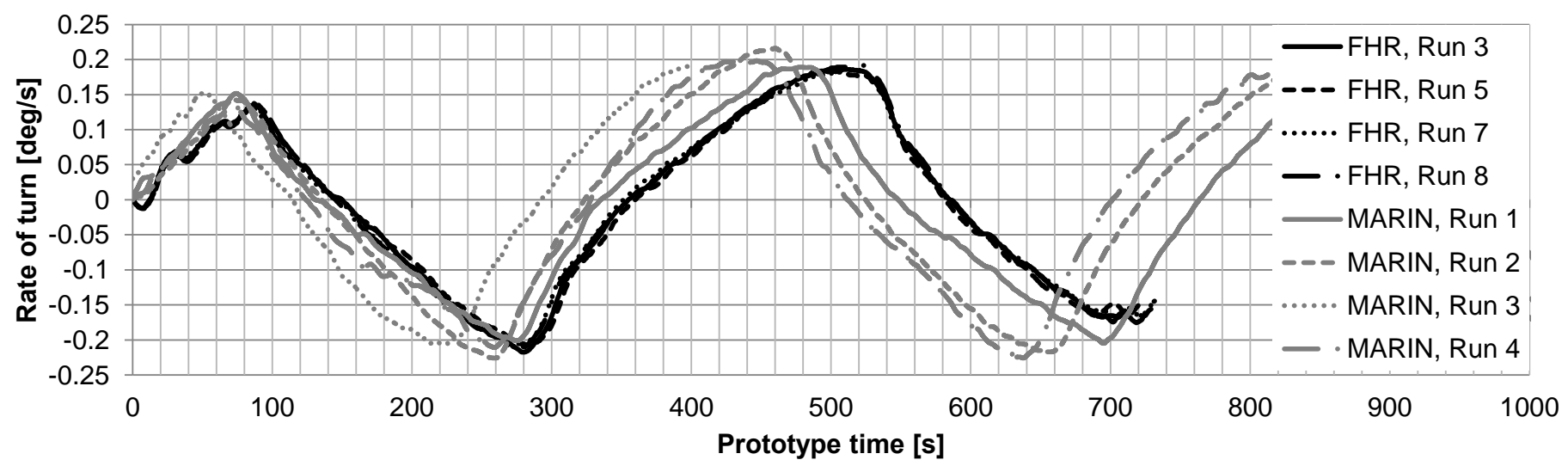

Figure 6 - Time dependence of the rate of turn for the $-20 /+5$ zigzag manoeuvres (20\% UKC) with KVLCC2 through free-running tests at FHR and MARIN

\section{MATHEMATICAL MODELS}

Mathematical models predicting the shallow water behaviour according to MARIN and FHR for the KVLCC2 are based on a different background. In this chapter the model from FHR will be described thoroughly. The model of MARIN (called MPP) can be found in [4] and is summarized as:

- An empirical method is used for the prediction of each module using a database of large parameter variance. No information from model tests is necessary to make the manoeuvring predictions.

- A modular type 4 DOF mathematical model is used with a physical description of the hull, propeller and rudder contributions. A mathematical model is derived for each under keel clearance.

- The slender body and cross flow drag theory is used for the predictions of the hull forces and uses the hull form of the considered ship model.

- The propeller and rudder forces are calculated using their characteristics. The coefficients of the respective mathematical models are directly calculated from the database.

Based on the captive tests, carried out in shallow water with the KVLCC2 in the towing tank at FHR, the coefficients of a tabular mathematical model were determined for each under keel clearance (Table 2). The model for the KVLCC2 is a $3+1$ DOF model with 3 coupled DOF for the horizontal motions and 1 for including roll.

\subsection{HULL FORCES}

\section{Velocity derivatives}

The hull forces are expressed as functions of the hydrodynamic angles (horizontal speed combinations):

$$
\begin{gathered}
\beta=\arctan \left(\frac{-v}{u}\right) \\
\gamma=\arctan \left(\frac{0.5 r L}{u}\right) \\
\chi=\arctan \left(\frac{0.5 r L}{v}\right)
\end{gathered}
$$

leading to the following velocity dependent equations:

$$
\begin{gathered}
X_{H}=\frac{1}{2} \rho L T\left[\left(u^{2}+v^{2}\right) X^{\prime(\beta)}+\left(u^{2}+\left(\frac{1}{2} r L\right)^{2}\right) X^{\prime(\gamma)}+\right. \\
\left.\left(v^{2}+\left(\frac{1}{2} r L\right)^{2}\right) X^{\prime}(\chi)\right] \\
Y_{H}=\frac{1}{2} \rho L T\left[\left(u^{2}+v^{2}\right) Y^{\prime(\beta)}+\left(u^{2}+\left(\frac{1}{2} r L\right)^{2}\right) Y^{\prime(\gamma)}+\right. \\
\left.\left(v^{2}+\left(\frac{1}{2} r L\right)^{2}\right) Y^{\prime}(\chi)\right] \\
K_{H}=\frac{1}{2} \rho L T^{2}\left[\left(u^{2}+v^{2}\right) K^{\prime(\beta)}+\left(u^{2}+\left(\frac{1}{2} r L\right)^{2}\right) K^{\prime(\gamma)}\right. \\
\left.\quad+\left(v^{2}+\left(\frac{1}{2} r L\right)^{2}\right) K^{\prime}(\chi)\right] \\
\quad+\left[K_{p}-|\varphi| \sqrt{\Delta \overline{G M}_{T}\left(\left(-K_{\dot{p}}+I_{x x}\right)\right)}\right] p+K_{u p} u p \\
N_{H}=\frac{1}{2} \rho L^{2} T\left[\left(u^{2}+v^{2}\right) N^{\prime(\beta)}+\left(u^{2}+\left(\frac{1}{2} r L\right)^{2}\right) N^{\prime(\gamma)}+\right. \\
\left.\left(v^{2}+\left(\frac{1}{2} r L\right)^{2}\right) N^{\prime}(\chi)\right]
\end{gathered}
$$

In the above equations the functions $X^{\prime(\beta)}, \ldots N^{\prime}(\chi)$ are expressed as tables in function of the given angle.

In Figure 7 the drift angle dependent tables are shown for the KVLCC 2 at $80 \%$ UKC. The tables are not made symmetrical although the purely hull dependent coefficients could be considered as being symmetrical. Linear interpolation is used for drift angles in between the tested values so that a close relationship exists between the test program and the accuracy of the tabular models. Yawing moments due to drift usually reach a maximum value at a drift angle range of 45 to 60 degrees; however, no tests have been executed in this range in the frame 
of the SIMMAN test program, which focused on small drift angles.

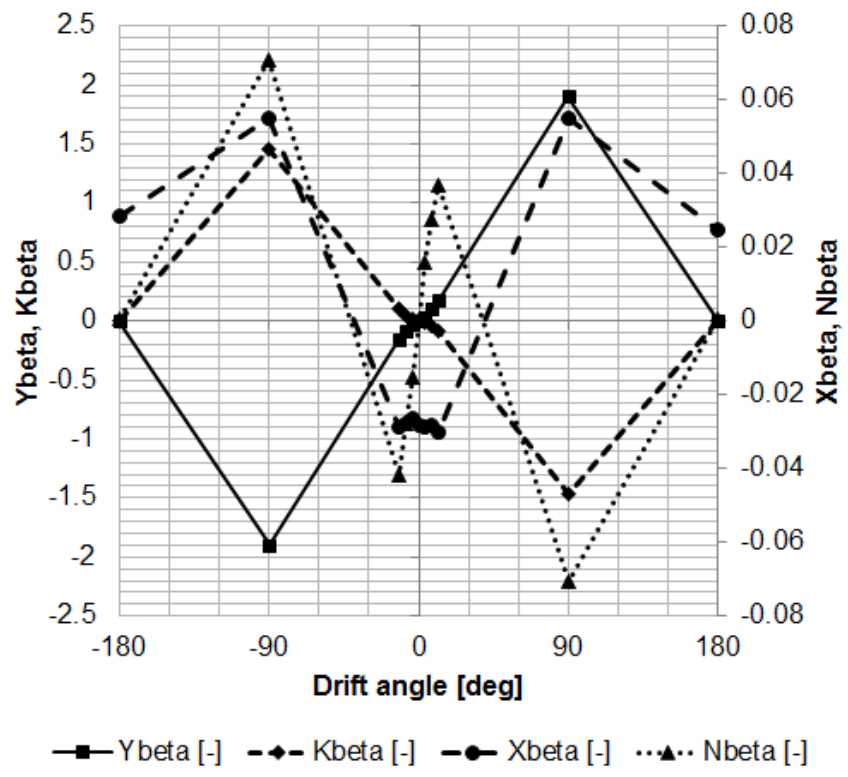

Figure 7 - Drift angle dependent tables for longitudinal and lateral force and yawing and roll moment

\section{Acceleration and centrifugal terms}

The acceleration and centrifugal terms are:

$$
\begin{gathered}
X_{I C}=\left(X_{\dot{u}}-m\right) \dot{u}+m v r+m x_{G} r^{2}-m z_{G} p r \\
Y_{I C}=\left(Y_{\dot{v}}-m\right) \dot{v}+\left(Y_{\dot{p}}+m z_{G}\right) \dot{p}+\left(Y_{\dot{r}}-m x_{G}\right) \dot{r}-m u r \\
K_{I C}=\left(K_{\dot{v}}+m z_{G}\right) \dot{v}+\left(K_{\dot{p}}-I_{x x}\right) \dot{p}+\left(K_{\dot{r}}+I_{x z}\right) \dot{r}+m z_{G} u r \\
N_{I C}=\left(N_{\dot{v}}-m x_{G}\right) \dot{v}+\left(N_{\dot{p}}+I_{x z}\right) \dot{p}+\left(N_{\dot{r}}-I_{z Z}\right) \dot{r}+m x_{G} u r
\end{gathered}
$$

The SIMMAN benchmark set for the KVLCC2 did not contain a test with varying longitudinal acceleration; for that reason the value of $X_{\dot{u}}$ was assumed to be $10 \%$ of the ship's mass.

The sway acceleration dependent terms could be derived from the results of the harmonic sway test. The harmonic yaw tests were used to determine the yaw acceleration dependent terms. The latter are also a tabular function of the drift angle.

The roll velocity and acceleration components have to be found by a roll decay test, which was not included in the SIMMAN benchmark data for the KVLCC2. So far FHR did not have the opportunity to perform a roll decay test. For that reason, the roll dependent derivatives will be zero during the simulations. The coupling between the roll and the other degrees of freedom is zero, because $\mathrm{KG} \approx \mathrm{T}$ and $\mathrm{I}_{\mathrm{xz}} \approx 0$. As such the model will rather be 3 DOF instead of 4 DOF.

\subsection{PROPULSION}

The propeller thrust is modelled by determining the wake factor in:

$$
\varepsilon=\arctan \left(\frac{\left(1-w_{T}\right) u}{0.7 \pi n D_{P}}\right)
$$

so that the thrust can be predicted as follows:

$$
T_{P}=\frac{0.7^{2}}{8} \pi^{3} \rho n^{2} D_{P}^{4} C_{T}(\varepsilon)\left(1+\tan ^{2} \varepsilon\right)
$$

in which $\mathbf{C}_{\mathbf{T}}$ is an alternative formulation of $\mathbf{K}_{\mathrm{T}}$, suitable for operations in four quadrants. The propeller's thrust in open water was determined for both ships. For the KVLCC2 the open water characteristic is comparable to the open water characteristic of the HMRI model available on the SIMMAN website [5].

The wake factor is expressed as a function of the propeller loading. For the KVLCC2 all benchmark tests were conducted at self-propulsion in the first quadrant, so a single value for the wake was determined for each under keel clearance.

The propeller shaft torque has not been modelled for the KVLCC2 due to the assumption of constant rpm.

\subsection{PROPULSION INDUCED FORCES}

For the KVLCC2 some assumptions have been made. As all tests were carried out at self-propulsion it is difficult to assess the actual influence of the propeller on the total forces. Therefore it was decided to include the propulsion component in the hull force for the sway, yaw and heel modes. For the surge component a constant thrust deduction factor was derived, based on the comparison between the result of resistance tests with the required thrust for self-propulsion:

$$
X_{P}=(1-t) T_{P}
$$

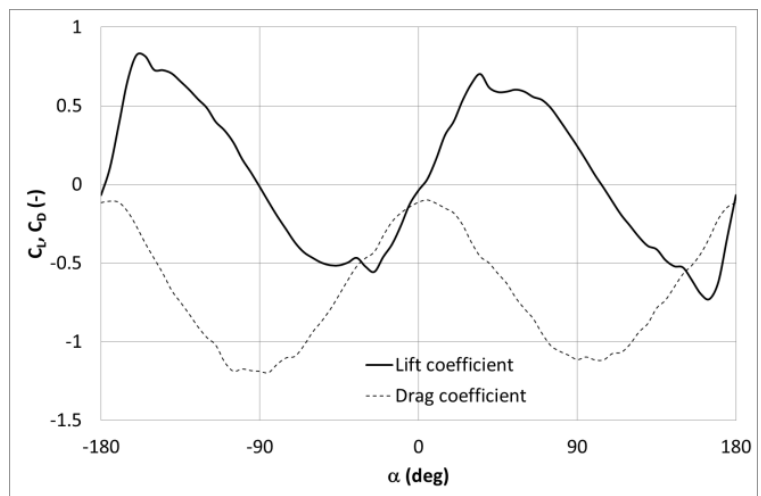

Figure 8 - Measured lift and drag on the rudder of the KVLCC2 in open water

\subsection{FORCES ACTING ON THE RUDDER}

Similar to the propeller's thrust the forces acting on the rudder are based on a model of the wake factor. To do so the 
open water lift and drag of the rudder have to be available. FHR performed open water tests with the rudders of the KVLCC2 for a variety of inflow angles $\alpha$ (Figure 8).

When the rudder is behind the ship the inflow is affected by the effective rudder angle and the drift near the rudder:

$$
\alpha=\delta+\delta_{0}+\beta_{R}
$$

$\delta_{0}$, the rudder angle for which the normal force $F_{N}$ acting on the rudder vanishes, is a correction for flow asymmetry:

$$
\delta_{0}=-\delta\left(F_{N}=0\right)
$$

$\beta_{R}$ is the local drift angle at the rudder:

$$
\beta_{R}=\arctan \left(\frac{-v_{R}}{u_{R}}\right)
$$

$u_{R}, v_{R}$ being the longitudinal and transverse component of the flow velocity near the rudder:

$$
V_{R}=\sqrt{u_{R}^{2}+v_{R}^{2}}
$$

In this way the forces on the rudder can be expressed as:

$$
\begin{gathered}
F_{X}=\frac{1}{2} \rho A_{R} V_{R}^{2}\left[C_{L} \sin \beta_{R}+C_{D} \cos \beta_{R}\right] \\
F_{Y}=\frac{1}{2} \rho A_{R} V_{R}^{2}\left[C_{L} \cos \beta_{R}-C_{D} \sin \beta_{R}\right]
\end{gathered}
$$

The problem is then to predict the velocity components near the rudder. The lateral component is believed to be:

$$
v_{R}=v+r x_{R}
$$

whereas the longitudinal component is affected by both the propeller flow and the hull. The used expression is based on the impulse theory.

$=\frac{1-w_{R}}{1-w_{T}} \sqrt{\left\{\begin{array}{c}\left.\left\{(1-k) \sin \varepsilon+k \sqrt{C_{T}+\sin ^{2} \varepsilon}\right]^{2}+(1-\eta) \sin ^{2} \varepsilon\right\} \\ \left\{\left[\left(1-w_{T}\right) u\right]^{2}+\left[0.7 \pi n D_{P}\right]^{2}\right\}\end{array}\right.}$

The wake factor $w_{R}$ in eq. 22 can be expressed as a tabular function of the rudder angle. A different function is necessary for the longitudinal and the lateral rudder force. The wake factor is also affected by the under keel clearance.

\subsection{RUDDER INDUCED FORCES}

Once the hull and propeller induced forces are computed the remaining fraction of the measured forces is used to compute the rudder induced part with the following equations:

$$
\begin{gathered}
X_{R}=\left(1-t_{R}\right) F_{X} \\
Y_{R}=\left(1+a_{H}\right) F_{Y} \\
K_{R}=-\left(z_{R}+a_{H} z_{H}\right) F_{Y} \\
N_{R}=\left(x_{R}+a_{H} x_{H}\right) F_{Y}
\end{gathered}
$$

In the above expressions the parameters were determined as a constant value for each under keel clearance.

\section{VALIDATION AT MODEL SCALE}

The goal of the SIMMAN workshop and the SimVal project is to give more insight in

- Verification and Validation of Ship Manoeuvring Simulation Methods with focus on model scale validation in SIMMAN;

- Sea Trials and Model Tests for Validation of Shiphandling Simulation Models with focus on full scale validation in SimVal.

Although both international research groups use model tests, the SimVal project extends the validation to the behaviour of the ships at full scale while the SIMMAN workshop remains at model scale with the benchmark ship models of which no full scale versions are available. The validation at model scale, discussed in this chapter, nevertheless shows that especially in shallow water, more insight is required to obtain at least comparable results at model scale.

The validation at model scale is based on a comparison of the time series and the trial characteristics (e.g. overshoot angles for zigzag manoeuvres) of the free-running model tests (FRMT) and the simulated results from the mathematical models (PMM for FHR and indicated by EMP for MARIN's empirical prediction program MPP).

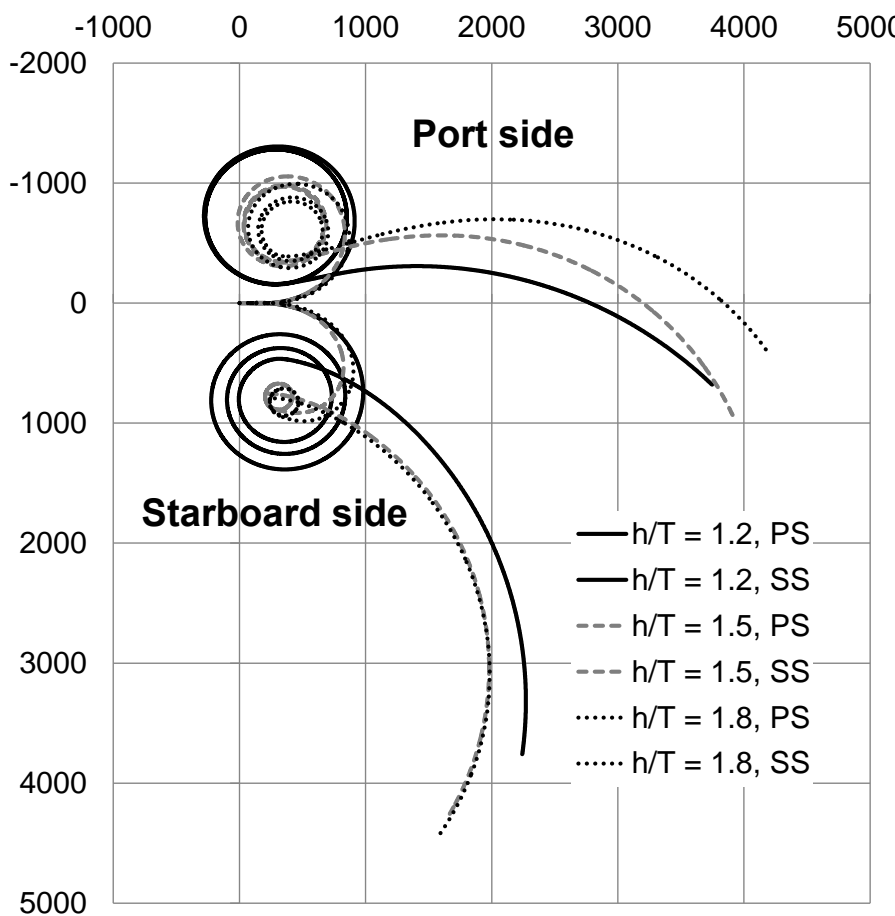

Figure 9 - Simulated 1080 degrees turning circles with pull-out manoeuvres with KVLCC2 based on the PMM tabular models of FHR 


\subsection{FROM DEEP TO SHALLOW WATER}

The simulated predictions of 1080 degrees turning circles followed by pull-out manoeuvres based on the PMM tabular model of FHR in medium deep to shallow water are shown in Figure 9. The tracks show the predictive power of the simulation models for decreasing water depth to draft ratio $\mathrm{h} / \mathrm{T}$ but reveal also some drawbacks of the simulation models:

- A (large) difference is observed between the starboard and port turn as the tabular models are not made symmetrical for the hull contribution and the single propeller asymmetry is thus not only found in the propeller dependent modules.

- The tabular models should be derived from an extensive test program of 300 tests including low speed and high drift manoeuvring. The unrealistic small starboard turning circles at 50\% and $80 \%$ UKC are due to the drop down of the forward speed to zero. A tuning of the longitudinal force $X^{\prime}(\chi)$ which was set to zero solves the drop down but increases the turning circle diameters for all UKCs. Regression models which describe the velocity dependent terms in polynomial expansions are widely used for the prediction of ship manoeuvring in limited operational conditions such as the well-known MMG model and other wholeship models. Because of the restricted number of coefficients, they are generally less accurate in a larger range of kinematic parameters, but on the other hand they offer the advantage of robustness. Tabular models that are derived from a limited number of test conditions should therefore be adapted to the test program and the expected values from full verified tabular models.
As only partial free-running turning circles were available from model tests a comparison must be treated with caution and is discussed in chapter 4.2 for the turning circles at $20 \%$ UKC.

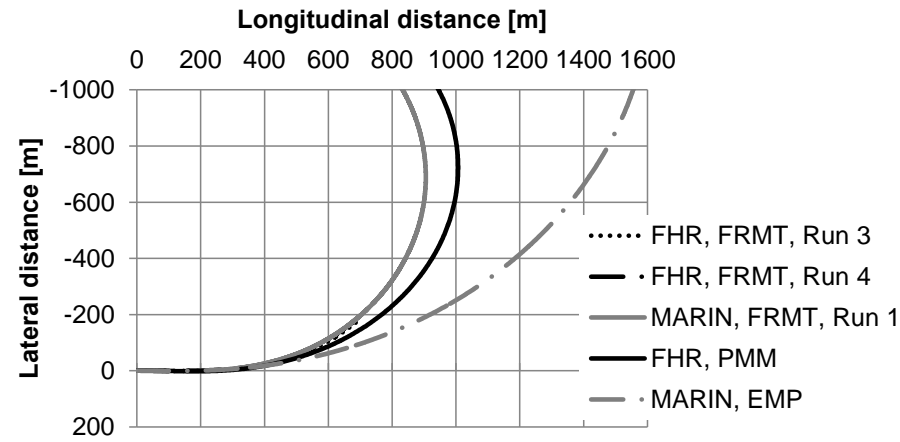

Figure 10 - Comparison of the simulated and measured tracks of the partial turning circle at $20 \%$ UKC for the KVLCC2

\subsection{TURNING CIRCLES at $20 \%$ UKC}

The comparison of the simulated and measured tracks of the partial turning circle at $20 \%$ UKC is shown in Figure 10. The PMM based tabular model of FHR gives an advance which is closer to the advance values measured during the freerunning tests at MARIN and FHR.

None of the empirical or tabular models predict the overshoot in the rate of turn and the final value of $-0.25 \mathrm{deg} / \mathrm{s}$ in Figure 11. The smallest discrepancy is in correspondence with the track found for the PMM based tabular model. For the time dependence of the drift angle (Figure 12) the empirical model predicts correctly the steady state while the increasing drift angle with time for the PMM model, even if steady state is expected, is due to the decreasing forward speed which was even more clearly observed for 50 and $80 \%$ UKC.

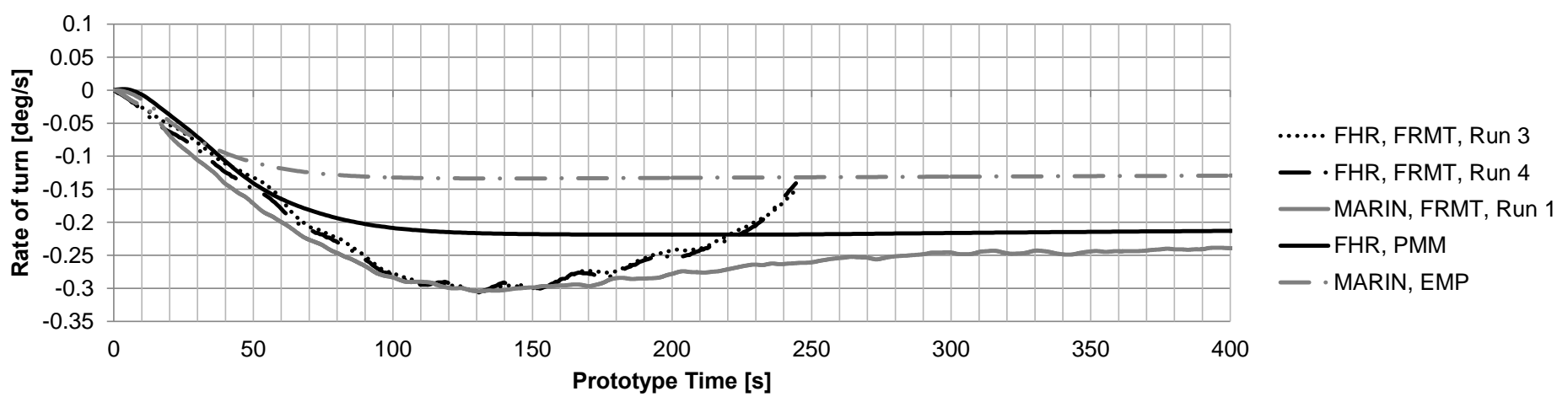

Figure 11 - Comparison of the simulated and measured time series of the rate of turn for the partial turning circle with KVLCC2 at $20 \%$ UKC 


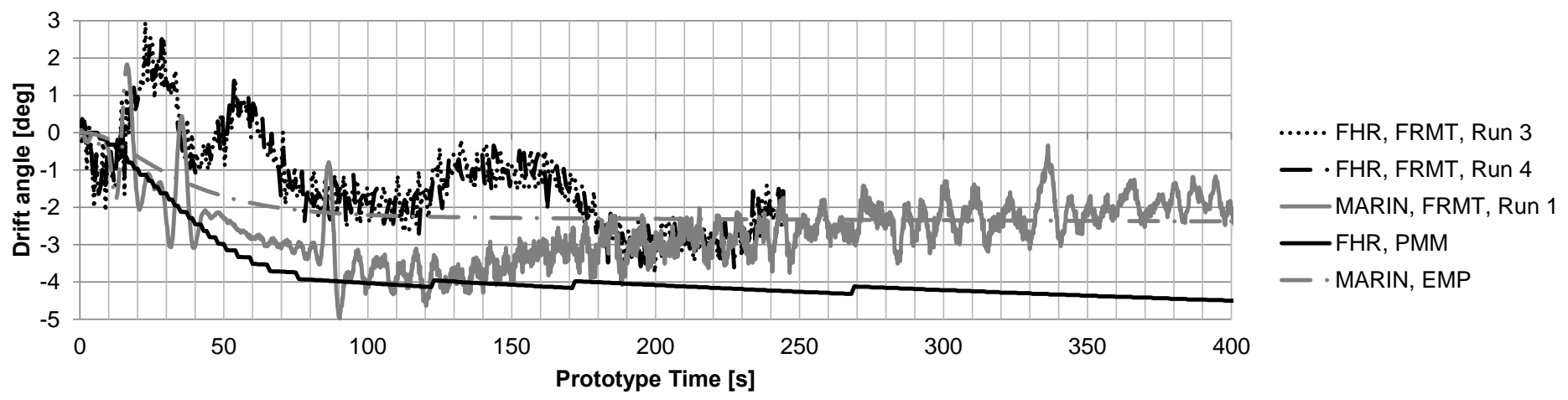

Figure 12 - Comparison of the simulated and measured time series of the drift angle for the partial turning circle with KVLCC2 at $20 \%$ UKC
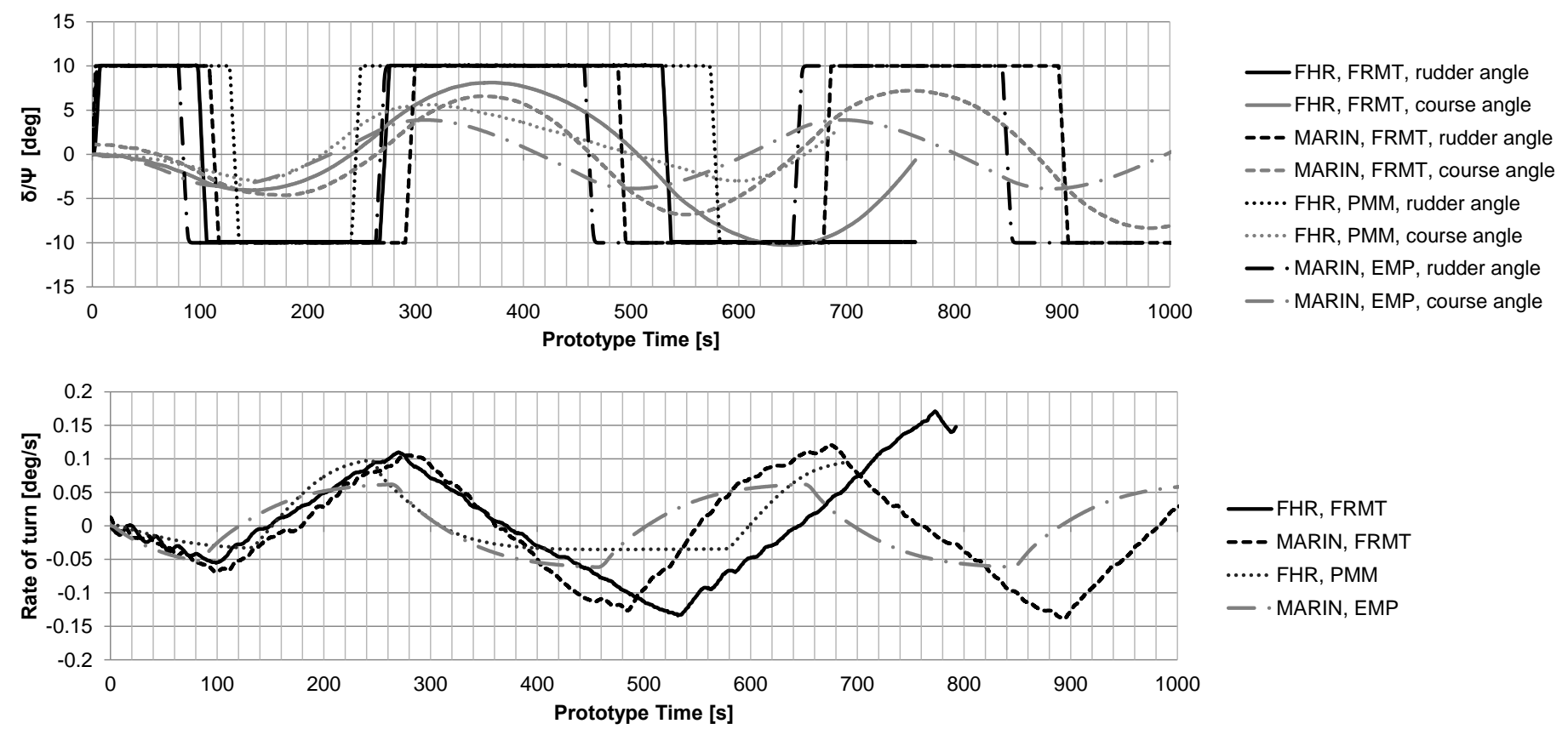

Figure 13 - Comparison of the simulated and measured time series for the $+10 /-2.5$ zigzag manoeuvre with KVLCC2 at $20 \%$ UKC
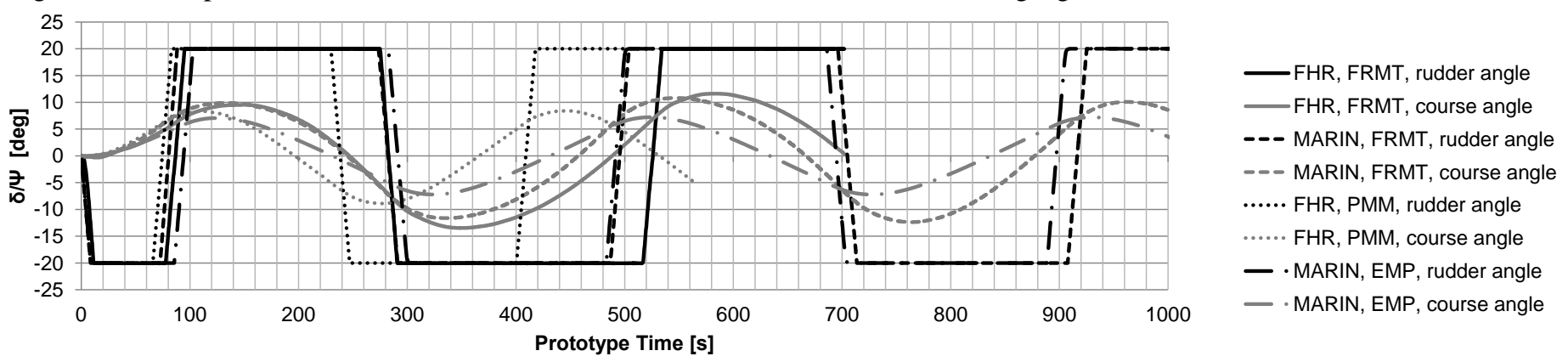


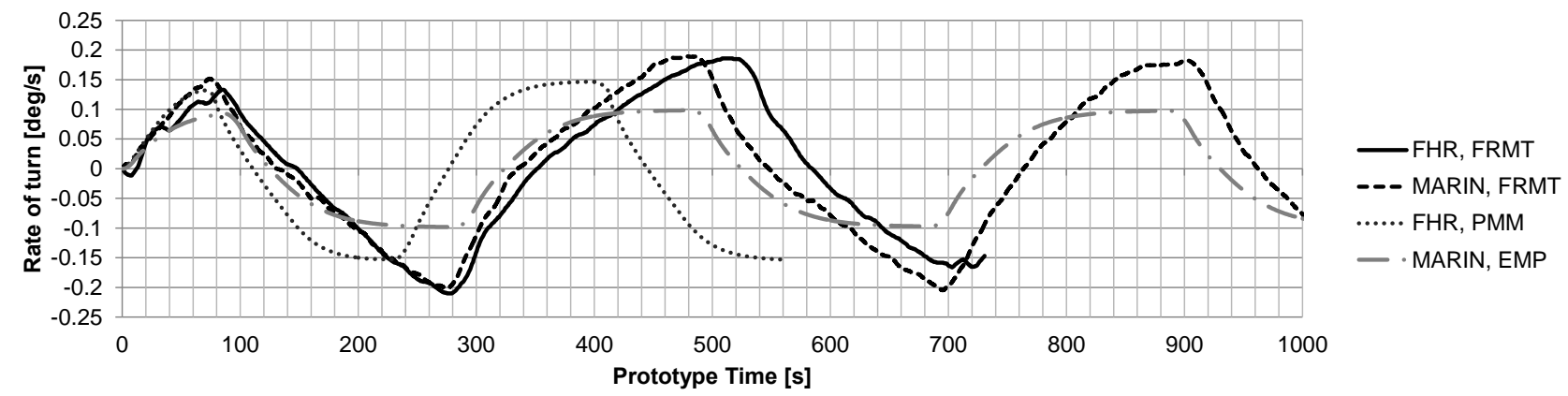

Figure 14 - Comparison of the simulated and measured time series for the $-20 /+5$ zigzag manoeuvre with KVLCC2 at $20 \%$ UKC

\section{CONCLUSIONS}

The prediction of the manoeuvring behaviour of ships in shallow water needs more attention from researchers involved in ship hydrodynamics. A first attempt was successfully tackled with the second SIMMAN workshop held in December 2014 in Denmark. Although the number of contributions of both model test executions and system based and numerical based predictions was still limited, following important remarks and conclusions can be made:

- Before completing the validation from model to full scale a first focus on the validation at model scale helps in defining the differences already observed between measurements and predictions at model scale.

- For the comparison of the predictions the availability of model test results of both captive and free-running manoeuvring tests is important. The accuracy of the tank facility and the test procedure has a major influence on the time dependence and the characteristics of free-running turning circle and zigzag manoeuvres. Although the initial conditions could be set by the carriage in the free-running model tests at FHR, the vicinity of the tank walls probably influenced the partial turning circles and larger accuracy is obtained for 20/5 compared to $10 / 2.5$ zigzag manoeuvres. The small angle of 2.5 degrees gives a larger spread in the decision making of the next rudder execute.

- As the free-running tests at MARIN and FHR were executed with the same fully appended model of the KVLCC2 (with a slighty different propeller), no variations in scale and rudder characteristics must be accounted for. Based on the time series results and tracks corresponding tests were selected and compared with the system based predictions.

- For the turning circle manoeuvres the PMM based tabular model at $20 \%$ UKC predicts better the advance and 90 degrees course change but problems occur as the forward speed drops to zero for the starboard turn at 50 and $80 \%$ UKC. The tabular model should be adapted to meet the restrictions induced by the test program. The empirical model overestimates the turning circle characteristics considerably for the KVLCC2.

- For the zigzag manoeuvres the correspondence in time variance between the model tests and simulations is better for the 20/5 zigzag test compared to the $10 / 2.5$ test. Nevertheless the mathematical models predict a more course stable KVLCC2 in $20 \%$ UKC with smaller overshoot angles than measured during the free-running tests.

As no completely satisfactory result is noticed for the validation at model scale both model testing and mathematical modelling should be further examined to detect the reasons for the differences. Flow measurements in captive and free-running tests could help in understanding the steady or transient behaviour of the water flow in the tank. These flows could further be compared with flow predictions from RANS calculations using CFD techniques so that the specific hydrodynamics in shallow water are better understood. During the SIMMAN workshop promising results have been shown concerning flow predictions so that further collaboration in model testing and prediction will bring the predictive power of simulation models closer to the real world.

\section{NOMENCLATURE}

$A_{R} \quad$ rudder area $\quad \mathrm{m}^{2}$

$C_{D} \quad$ drag coefficient -

$C_{L} \quad$ lift coefficient -

$C_{T} \quad$ thrust coefficient -

$D_{P} \quad$ propeller diameter m

$F_{X} \quad$ longitudinal rudder force $\quad \mathrm{N}$

$F_{Y} \quad$ lateral rudder force N

$h \quad$ water depth m

$h_{\max } \quad$ maximum water depth m 


$\begin{array}{lll}I_{* *} & \text { moment or product of inertia } & \mathrm{kgm}{ }^{2} \\ K & \text { roll moment } & \mathrm{Nm} \\ k & \sim \text { distance propeller }- \text { rudder } & - \\ \mathrm{L} & \text { length between perpendiculars } & \mathrm{m} \\ \mathrm{L} & \text { lenthe of towing tank/basin } & \mathrm{m} \\ m & \text { ship's mass } & \mathrm{kg} \\ N & \text { yaw moment } & \mathrm{Nm} \\ n & \text { propeller rate } & 1 / \mathrm{s} \\ p & \text { roll velocity } & \mathrm{deg} / \mathrm{s} \\ r & \text { yaw velocity } & \mathrm{deg} / \mathrm{s} \\ \mathrm{T} & \text { draft } & \mathrm{m} \\ T_{P} & \text { propeller thrust } & \mathrm{N} \\ \mathrm{t} & \text { thrust deduction factor } & - \\ \mathrm{t}_{\mathrm{R}} & \text { rudder deduction factor } & - \\ u & \text { longitudinal ship velocity } & \mathrm{m} / \mathrm{s} \\ v & \text { lateral ship velocity } & \mathrm{m} / \mathrm{s} \\ u_{R} & \text { longitudinal velocity near rudder } & \mathrm{m} / \mathrm{s} \\ v_{R} & \text { lateral velocity near rudder } & \mathrm{m} / \mathrm{s} \\ X & \text { longitudinal force } & \mathrm{N} \\ x_{G} & \text { longitudinal centre of gravity } & \mathrm{m} \\ x_{R} & \text { longitudinal position of rudder } & \mathrm{m} \\ Y & \text { sway force } & \mathrm{N} \\ \mathrm{W} & \text { width of towing tank/basin } & \mathrm{m} \\ w_{R} & \text { wake factor for the rudder } & - \\ w_{T} & \text { wake factor for the thrust } & - \\ z_{G} & \text { vertical centre of gravity } & \mathrm{m} \\ z_{R} & \text { vertical position of rudder } & \mathrm{m} \\ & & \mathrm{kg} / \mathrm{m}^{3} \\ \alpha & \text { inflow angle } & \mathrm{deg} \\ \beta & \text { drift angle } & \mathrm{deg} \\ \beta_{R} & \text { drift angle near rudder } & \mathrm{deg} \\ \gamma & \text { yaw angle } & \mathrm{deg} \\ \Delta & \text { displacement } & \mathrm{deg} \\ \delta & \text { rudder angle } & \mathrm{N} \\ \delta_{0} & \text { rudder asymmetry correction } & \mathrm{deg} \\ \varepsilon & \text { propeller loading angle } & \mathrm{deg} \\ & \text { propeller diameter } \div \text { rudder height } & - \\ & \text { water density } & \text { heel angle } \\ & & \end{array}$
$\chi \quad$ yaw-drift correlation angle $\quad$ deg
EMP Empirical
FHR Flanders Hydraulics Research
ITTC International Towing Tank Conference
MARIN Maritime Research Institute the Netherlands
MMG Manoeuvring Mathematical Model Group
MPP Mannoevring Prediction Program
PMM Planar Motion Mechanism

\section{ACKNOWLEDGMENTS}

The authors wish to acknowledge the organizing committee of the SIMMAN workshop 2014 which has been held in Lyngby, Denmark from 8 to 10 December 2014. The discussions during the workshop have revealed further considerations and attentions in model testing and simulation. This paper is partly based on the work executed by FHR and MARIN in the framework of SIMMAN.

\section{REFERENCES}

[1] Van Kerkhove, G., Vantorre, M. \& Delefortrie, G., 2009. Advanced Model Testing Techniques for Ship Behaviour in Shallow and Confined Water. Proceedings of The First International Conference on Advanced Model Measurement Technology for the EU Maritime Industry, p.29.

[2] PIANC, 1992. Capability of ship manoeuvring simulation models for approach channels and fairways in harbours: Report of Working Group no. 20 of Permanent Technical Committee II, Supplement to PIANC Bulletin, No. 77, 49 pp.

ITTC website http://ittc.info/downloads/Generel\%20files/pdfprocedu res2014/7.5-02-06-01.pdf $\&$ http://ittc.info/downloads/Generel\%20files/pdfprocedu res2014/7.5-02-06-02.pdf (2.1.1.4)

[4] Toxopeus, S.L. and Lee, S.W.; Comparison of manoeuvring simulation programs for SIMMAN test cases; SIMMAN 2008 Workshop on Verification and Validation of Ship Manoeuvring Simulation Methods, 2008

[5] http://www.simman2014.dk/ 